\title{
Artigo/Article
}

\section{Caracterização molecular de Pseudomonas aeruginosa resistentes a carbapenêmicose produtoras de metalo- $\beta$-lactamase isoladas em hemoculturas de crianças e adolescentes com câncer}

\author{
Molecular characterization of carbapenem-resistant and metallo- $\beta$-lactamase-producing \\ Pseudomonas aeruginosa isolated from blood cultures from children and teenagers with cancer
}

\author{
Thais Ávila Fernandes ${ }^{1}$, Carlos Alberto Pires Pereira ${ }^{2}$, Antonio Sergio Petrili ${ }^{2}$ e Antônio Carlos Campos Pignatari ${ }^{1}$
}

\begin{abstract}
RESUMO
Introdução: $\mathrm{O}$ objetivo do estudo foi avaliar a prevalência e a disseminação de amostras de Pseudomonas aeruginosa resistente aos carbapenêmicos e produtoras de metalo- $\beta$-lactamases isoladas de hemoculturas (2000-2005) de pacientes do Instituto de Oncologia Pediátrica da UNIFESP (IOP-GRAACC). Métodos e Resultados: Cinquenta e seis amostras de Pseudomonas aeruginosa foram isoladas de 49 pacientes. Trinta e duas dessas amostras foram classificadas como resistentes aos carbapenêmicos pela técnica de disco difusão e submetidas a reação de PCR para detecção de genes de MBL. Dezoitos dessas 32 amostras evidenciaram o gene $b$ la $_{\mathrm{SPM}-1}$. Oito amostras selecionadas em diferentes anos no período de estudo apresentaram o mesmo perfil genético por pulsed-field gel electrophoresis. A terapêutica antimicrobiana foi considerada adequada em apenas $23,5 \%$ dos pacientes com bacteremia por $P$. aeruginosa carreando $b l a_{\mathrm{SPM}-1}$ e letalidade de 70,6\% no período de até 30 dias após a bacteremia e uma inadequação inicial dos esquemas antibióticos utilizados Conclusões: Evidenciamos a presença de um clone de $P$. aeruginosa resistente aos carbapenêmicos carreando $b{ }_{1} a_{\mathrm{SPM}-1}$ que persistiu em amostras de hemocultura pelo período de 6 anos na instituição, com alta letalidade, justificando uma vigilância epidemiológica rigorosa e uma readequação dos esquemas de terapia antimicrobianos na instituição.
\end{abstract}

Palavras-chaves: Pseudomonas aeruginosa. Metalo- $\beta$-lactamase. Câncer. Hemocultura. Infecção hospitalar.

\begin{abstract}
Introduction: The objective of this study was to evaluate the prevalence and dissemination of carbapenem-resistant and metallo- $\beta$-lactamase-producing Pseudomonas aeruginosa isolated from blood-stream samples (2000-2005) that were collected from patients admitted to the Institute of Pediatric Oncology, UNIFESP (IOP-GRAACC). Methods and Results: Fifty-six $P$. aeruginosa samples were isolated from 49 patients. Thirty-two of these samples were classified as carbapenem-resistant using the disc diffusion method and were subjected to the PCR reaction in order to detect MBL genes. Eighteen of these 32 isolates showed the $b l a_{\mathrm{SPM}-1}$ gene. Eight samples selected in different years over the study period presented the same genetic profile according to pulsed-field gel electrophoresis. The antimicrobial therapy was considered adequate for only $23.5 \%$ of the patients with bacteremia due to $P$. aeruginosa carrying the $b l a_{\mathrm{SPM}-1}$ gene, and a high lethality rate of $70.6 \%$ was observed during the 30 -day period after bacteremia and an inadequate initial antibiotic regimen. Conclusions: We detected the presence of a clone of carbapenem-resistant $P$. aeruginosa carrying $b l a_{\mathrm{SPM}-1}$ that persisted in blood culture samples over a six-year period at the institution, with high lethality, thus justifying rigorous epidemiological surveillance and a rearrangement of the antimicrobial therapy regimens at the institution.
\end{abstract}

Key-words: Pseudomonas aeruginosa. Metallo- $\beta$-lactamase. Cancer. Blood culture. Hospital infection.

1. Laboratório Especial em Microbiologia Clínica, Escola Paulista de Medicina, Universidade Federal de São Paulo, São Paulo, SP. 2. Instituto de Oncologia Pediátrica-Grupo de Apoio ao Adolescente e à Criança com Câncer, Escola Paulista de Medicina, Universidade Federal de São Paulo, São Paulo, SP.

Endereço para correspondência: Prof. Antonio Carlos Campos Pignatari. Disciplina de Infectologia UNIFESP/EPM. Rua Leandro Dupret 188. Vila Clementino, 04025-010 São Paulo, SP.

Tel: 5511 5081-2965

e-mail: info@lemc.com.br

Recebido para publicação em 12/11/2009

Aceito em 11/01/2010

\section{INTRODUÇÃO}

Infecções por bactérias Gram-negativas em ambiente hospitalar constituem grave problema de saúde pública, devido a sua frequência, morbidade, mortalidade e custo do tratamento. Pseudomonas aeruginosa é considerada um importante patógeno em ambiente hospitalar, comumente isolada em infecções de corrente sanguínea (ICS) e com frequência apresentam multirresistência aos antimicrobianos, inclusive aos carbapenêmicos (R).

No período estudado, observou-se um aumento de pacientes com ICS causada por P. aeruginosa no Instituto de Oncologia Pediátrica - Grupo de Apoio ao Adolescente e à Criança com Câncer (IOP-GRAACC), vinculado à Escola Paulista de Medicina da Universidade Federal de São Paulo (EPM-UNIFESP), especializado no tratamento de crianças e adolescentes com câncer. Nesse período, os pacientes apresentaram hemoculturas positivas para $P$. aeruginosa com perfil de resistência característica de cepas produtoras de metalo- $\beta$ lactamase $(\mathrm{MBL})$, na maioria resistentes a todos antimicrobianos com exceção da polimixina B. Posteriormente, foi confirmada a presença de MBL no Laboratório Especial em Microbiologia Clínica (LEMC) da EPM - UNIFESP com caracterização de uma nova enzima denominada SPM $^{1}$.

O presente estudo tem como objetivo avaliar a persistência desta cepa de $P$. aeruginosa carreando MBL de infecções de corrente sanguínea, sua disseminação através de tipagem molecular e as características clínicas e epidemiológicas.

\section{MÉTODOS}

Foram estudadas, retrospectivamente, amostras de ICS em crianças e adolescentes com câncer, admitidos no IOP-GRAACC, vinculado ao Departamento de Pediatria da EPM - UNIFESP, entre novembro de 2000 e dezembro de 2005. 
Os critérios de inclusão foram: presença de ICS e neutropenia; amostras isoladas somente de hemocultura; foram considerados óbitos até 30 dias após a bacteremia; os pacientes encontravam-se nos seguintes locais: unidade de terapia intensiva, ambulatório, enfermaria e setor de transplante de medula óssea. Os critérios abordados para definir a adequação do tratamento foram: adequado, inadequado e corrigido. $\mathrm{O}$ critério de exclusão foi: bacteremia do mesmo paciente em um intervalo inferior a um mês.

\section{Análise clínica e epidemiológica}

A análise epidemiológica e a avaliação clínica foram realizadas a partir de dados obtidos dos prontuários. Para isso, foi utilizada uma Ficha de Dados Clínicos contendo idade, sexo, data de internação e de coleta da hemocultura; diagnóstico de doença de base; local da internação; neutropenia; evolução do paciente (se o paciente foi a óbito até 30 dias após a bacteremia, o óbito foi considerado, caso contrário foi classificado como alta); sexo; fonte da bacteremia e terapia antimicrobiana utilizada.

\section{Microbiologia}

Durante o período de estudo, foram encaminhadas ao Laboratório Especial de Microbiologia Clínica (LEMC) 120 $P$. aeruginosa provenientes de hemocultura do IOP segundo protocolo de vigilância de hemoculturas pré-estabelecido sendo armazenadas em água destilada estéril a temperatura ambiente ou em TSB acrescido com $15 \%$ de glicerina a $-20^{\circ} \mathrm{C}$.

Os testes de sensibilidade aos antimicrobianos foram realizados através da técnica de disco difusão. Os discos de antimicrobianos (Oxoid', Basingstoke, Inglaterra) utilizados foram: aztreonam, imipenem, meropenem e polimixina B. Para o controle de qualidade, foram utilizadas as cepas de P. aeruginosa ATCC 27853 e Escherichia coli ATCC 25922. Os resultados foram interpretados de acordo com os critérios de sensibilidade estabelecidos pelo Clinical Laboratory Standard Institute (CLSI) para P. aeruginos $a^{2}$. A interpretação do halo da polimixina B foi baseada no estudo de Gales e cols ${ }^{3}$.

\section{Testes fenotípicos e genotípicos}

As amostras de $P$. aeruginosa confirmadas como resistentes aos carbapenêmicos foram submetidas ao teste de triagem para avaliação fenotípica da produção de MBL de acordo com Picão cols ${ }^{4}$. O controle positivo utilizado foi a amostra controle produtora de SPM1: P. aeruginosa $\mathrm{P} 1088$. Como controle negativo foi utilizado a cepa de $P$. aeruginosa ATCC 27853.

As amostras de $P$. aeruginosa detectadas fenotipicamente como produtoras de $\mathrm{MBL}$ foram investigadas quanto à presença dos genes $b l a_{\mathrm{SPM}-1}, b l a_{\mathrm{IMP}}, b l a_{\mathrm{VIM}-1}$ e $b l a_{\mathrm{VIM}-2}$ que codificam as MBL utilizando-se a técnica de reação da polimerase em cadeia (PCR) ${ }^{1}$.

Foram selecionadas para tipagem molecular pela técnica de pulsed-field gel eletrophoresis (PFGE) as amostras de P. aeruginosa resistentes a carbapenêmicos e confirmadas pela técnica de PCR como produtoras do gene $b l a_{\mathrm{SPM}-1}$ para comparação. Após digestão do DNA cromossômico pela enzima SpeI foi realizada eletroforese em gel de agarose a $1 \%$ no sistema CHEF-DR III (Bio-Rad, Richmond, CA, EUA). Foram incluídas 3 amostras controle isoladas entre novembro de 2000 e março de 2001. Os perfis moleculares pela técnica de PFGE foram analisados visualmente, seguindo o critério de Tenover e cols 5 .

Para análise da similaridade genética foi utilizado o sistema Bionumerics com construção de dendrograma considerando amostras pertencentes ao mesmo clone com até $4 \%$ de tolerância. Amostras com similaridade genética abaixo de $90 \%$, não são consideradas relacionadas.

\section{Ética}

Este estudo foi previamente submetido à avaliação e aprovação pelo Comitê de Ética em Pesquisa (CEP) da instituição.

\section{RESULTADOS}

Das 120 amostras de $P$. aeruginosa isoladas de hemoculturas e armazenadas no banco de microrganismos no LEMC, somente 56 amostras foram viáveis e utilizadas no estudo.

Entre as 56 amostras de P. aeruginosa analisadas, 24 (42,9\%) eram sensíveis aos carbapenêmicos e $32(57,1 \%)$ resistentes. Nas amostras resistentes aos carbapenêmicos pelo método de disco-difusão, foi realizado o teste fenotípico (disco aproximação) para detecção de MBL. Sensibilidade acima de $50 \%$ foi observada apenas para aztreonam $(73,2 \%)$ e polimixina B (100\%). Trinta e duas $(57,1 \%)$ amostras foram resistentes aos carbapenêmicos. Dezoito (56,2\%) amostras de $P$. aeruginosa foram classificadas como produtoras de MBL pelo teste de disco aproximação.

Dentre as 18 amostras de P. aeruginosa, inicialmente classificadas fenotipicamente como produtoras de $\mathrm{MBL}$, a presença do gene $b l a_{\mathrm{SPM}-1}$ foi confirmada em $18(100 \%)$ isolados. Nenhuma amostra apresentou produto de amplificação de PCR para os outros genes de MBL estudados $\left(b l a_{\mathrm{IMP}-1}, b l a_{\mathrm{VIM}-1}, b l a_{\mathrm{VIM}-2}\right)$. Dentre as 18 amostras produtoras do gene $b l_{\mathrm{SPM}-1}$, também, foi observada uma sensibilidade de $100 \%$ para aztreonam e polimixina B.

\section{Tipagem mlecular}

Para oito amostras produtoras de $b l a_{\mathrm{SPM}-1}$ isoladas em diferentes anos, foi realizada a técnica de tipagem molecular por (PFGE). No dendrograma (Figura 1) observamos que as oito amostras de P. aeruginosa $b_{\mathrm{SPM}-1}$ apresentaram uma similaridade genética acima de $98 \%$. Três amostras sensíveis aos carbapenêmicos, isoladas de hemoculturas de pacientes do IOP no mesmo período do estudo (P4515, P5126 e P5279) não demonstraram similaridade genética (menor que 90\%).

\section{Análise clínica e epidemiológica}

Analisando-se as características clínicas dos 49 pacientes portadores das 56 amostras de $P$. aeruginosa observa-se que a maioria $(44,9 \%)$ apresentava leucemia e o mesmo ocorre nos 17 pacientes das 18 amostras de $P$. aeruginosa produtoras do gene $b l a_{\mathrm{SPM}-1}(35,3 \%$ leucemias).A maioria (46,1\%) estava internada em enfermaria.Já entre as cepas de $P$. aeruginosa produtoras do gene $b l_{\mathrm{SPM}-1}$, a maioria $(41,2 \%)$ dos pacientes, estava na enfermaria em seguida no TMO $(35,3 \%)$. Quanto à fonte de bacteremia, foi primária em $55,8 \%$ e foi secundária em $44,2 \%$ no total das amostras analisadas. Das 18 amostras de P. aeruginosa produtoras do gene $b l_{\text {SPM-1 }}, 82,3 \%$ dos pacientes estavam neutropênicos. Quanto à fonte da bacteremia, foi primária em 53\% e secundária em $47 \%$ (Tabela 1).

O tratamento foi adequado em 46,1\%, inadequado em 17,3\% e corrigido em 34,6\% dos episódios de bacteremia. Nos 17 pacientes, dos quais foram isoladas as 18 amostras de $P$. aeruginosa produtoras de SPM, o tratamento foi adequado em $23,5 \%$, foi inadequado em $23,5 \%$ e corrigido em $53 \%$. Quanto à evolução clínica, 70,6\% dos 17 pacientes com $P$. aeruginosa produtora da MBL (gene $b l a_{\mathrm{SPM}-1}$ ) evoluíram a óbito (Tabela $\mathbf{1}$ ). 
TABELA 1 - Características clínicas e epidemiologia dos 49 pacientes com isolamento de $P$. aeruginosa em hemoculturas.

\begin{tabular}{|c|c|c|c|}
\hline Variável & $\begin{array}{c}\text { Total das } \\
\text { amostras estudadas }(\%)\end{array}$ & $\begin{array}{c}\text { Amostras resistentes aos } \\
\text { carbapenêmicos não produtoras de MBL (\%) }\end{array}$ & $\begin{array}{c}\text { Amostras resistentes aos } \\
\text { carbapenêmicos produtoras de MBL (\%) }\end{array}$ \\
\hline \multicolumn{4}{|l|}{ Gênero } \\
\hline feminino & 44,9 & 42,9 & 58,8 \\
\hline masculino & 55,1 & 57,1 & 41,2 \\
\hline \multicolumn{4}{|l|}{ Doença de Base } \\
\hline leucemias & 44,9 & 57,1 & 35,3 \\
\hline linfomas & 10,2 & 14,3 & 11,7 \\
\hline Tu SNC & 10,2 & 7,2 & 5,9 \\
\hline TMO & 12,2 & -- & 35,3 \\
\hline outros & 30,6 & 21,4 & 11,8 \\
\hline \multicolumn{4}{|l|}{ Local } \\
\hline UTI & 19,2 & 21,4 & 17,6 \\
\hline enfermaria & 46,1 & 57,1 & 41,2 \\
\hline ambulatório & 21,1 & 21,4 & 5,9 \\
\hline TMO & 15,4 & -- & 35,3 \\
\hline \multicolumn{4}{|l|}{ Neutropenia } \\
\hline não & 25,0 & 14,3 & 17.7 \\
\hline $\operatorname{sim}$ & 75,0 & 85,7 & 82,3 \\
\hline \multicolumn{4}{|c|}{ Fonte da bacteramia } \\
\hline primária & 55,8 & 35,7 & 53,0 \\
\hline secundária & 44,2 & 64,3 & 47,0 \\
\hline \multicolumn{4}{|l|}{ Tratamento } \\
\hline adequado & 46,1 & 35,7 & 23,5 \\
\hline inadequado & 17,3 & 28,6 & 23,5 \\
\hline corrigido & 34,6 & 35,7 & 53,0 \\
\hline \multicolumn{4}{|l|}{ Evolução } \\
\hline alta & 53,8 & 42,9 & 29,4 \\
\hline óbito & 46,2 & 57,1 & 70,6 \\
\hline
\end{tabular}

Tu SNC: tumor no sistema nervoso central, TMO: transplante de medula óssea, UTI: unidade de terapia intensiva.

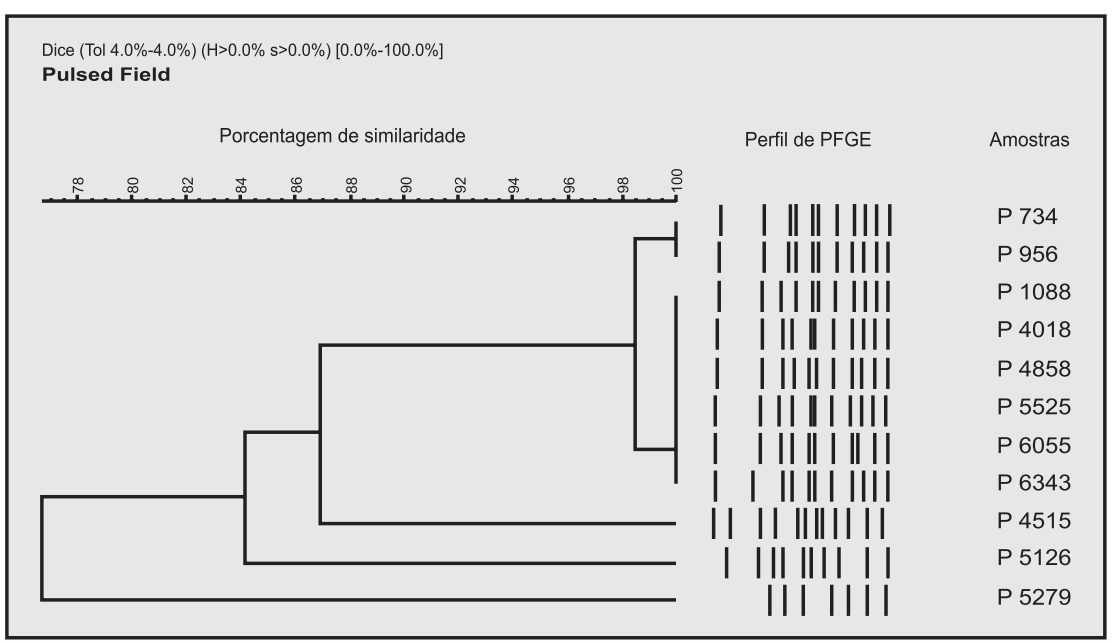

FIGURA 1 - Amostras analisadas: P734, P956, P1088, P4018, P4858, P5525, P6055, P6343, P4515, P5126, P5279.

\section{DISCUSSÃO}

Pseudomonas aeruginosa é o principal agente etiológico entre os bacilos Gram-negativos não-fermentadores de glicose e o quarto agente etiológico mais isolado em ICS. Infecção causada por esses microrganismos permanece como principal causa de óbito em pacientes oncológicos ${ }^{6,7}$.
As MBL adquiridas são codificadas por genes cassetes localizados tanto no cromossomo quanto no plasmídio bacteriano. No entanto, com exceção da enzima SPM-1 ${ }^{1,8}$, as demais MBLs adquiridas (IMP-1, VIM-1, VIM-2, SPM-1) são codificadas por genes localizados em integrons favorecendo a sua disseminação clonal ${ }^{9-12}$.

A prevalência de MBL entre as 32 amostras de $P$. aeruginosa resistentes aos carbapenêmicos provenientes de hemoculturas, avaliadas no presente estudo, foi de $59 \%$, todas produtoras de $b l a_{\mathrm{SPM}-1}$. 
Estudo realizado por Balan ${ }^{13}$, com 120 amostras de P. aeruginosa resistentes aos carbapenêmicos provenientes de diferentes sítios corpóreos isoladas no Hospital São Paulo, e no GRAACC, em 2004 (4 amostras), a prevalência de MBL foi de 38,1\%, sendo $20 \%$ de $b l a_{\mathrm{IMP}-1}$ e $80 \%$ de $b l a_{\mathrm{SPM}-1}$. Em nosso estudo, não detectamos a presença de $b l a_{\mathrm{IMP}-1}$.

O primeiro relato de SPM-1 foi feito por Toleman e cols em $2002^{1}$ em uma amostra clínica de $P$. aeruginosa isolada no IOP-GRAACC, em fevereiro de 2001. Esta amostra (P1088) faz parte deste estudo, e foi posteriormente encontrada em diferentes regiões brasileiras ${ }^{1,14}$.

Durante o estudo, observamos que a primeira amostra de P. aeruginosa produtora de SPM- 1 foi isolada em novembro de 2000 (amostra P700), portanto, anterior ao relatado de Toleman e cols ${ }^{1}$ demonstrando que a P. aeruginosa carreando $b l a_{\mathrm{SPM}-1}$ já estava presente na Instituição há pelo menos 1 ano.

Por PFGE observamos em 8 amostras que continham o gene $b l a_{\mathrm{SPM}-1}$, o mesmo perfil caracterizando um clone ${ }^{5}$. Essas amostras foram isoladas em diferentes anos, demonstrando que esse clone permanece nesse hospital e é o mesmo que o encontrado em diferentes regiões brasileiras ${ }^{14}$.

A resistência aos carbapenêmicos em $P$. aeruginosa pode estar associada a outros mecanismos além da produção de MBL. Marra ${ }^{15}$ observou que $81,1 \%$ das amostras de $P$. aeruginosa isoladas de ICS resistentes aos carbapenêmicos possuíam MBL. Já Balan ${ }^{13}$, em 2007 , observou que $62,5 \%$ das amostras de $P$. aeruginosa isoladas de diferentes sítios eram resistentes aos carbapenêmicos e não produziam MBL. A diminuição da permeabilidade de membrana externa (porinas) e/ou a hiperexpressão de bombas de efluxo, podem ser os prováveis mecanismos responsáveis por esse fenótipo de resistência.

A neutropenia está intimamente ligada ao processo e ao seu tratamento, sendo o principal fator de risco para complicações infecciosas nesses pacientes. Para diminuir a morbimortalidade relacionada ao processo infeccioso é muito importante a administração imediata de antibioticoterapia empírica de amplo espectro. No presente estudo, $75 \%$ dos 49 pacientes estudados e $83,3 \%$ dos 17 pacientes com infecção por $P$. aeruginosa produtora de MBL (SPM1) estavam neutropênicos. Entretanto, a terapêutica foi adequada em apenas 46,1\%. Quando consideramos apenas pacientes com amostras produtoras de MBL a adequação foi ainda menor (27,7\%). Considerando a elevada prevalência de MBL particularmente $b l a_{\mathrm{SPM}-1}$ nas amostras isoladas dos pacientes do IOP e uma elevada taxa de mortalidade, a terapêutica empírica em episódios de bacteremia nessa população de pacientes poderia incluir as polimixinas pelo menos nos pacientes de elevado risco infeccioso (leucemias, TMO e/ou com períodos de neutropenia e internação prolongados).

Para essa população de pacientes de alto risco o tempo entre coleta e identificação é muito elevado, associando-se a inadequação da terapêutica e contribuindo para uma maior mortalidade. A detecção precoce de metalo enzimas por métodos moleculares poderá contribuir para um resultado mais rápido utilizando PCR multiplex em tempo real com primers para as enzimas IMPs, VIMs, SPM-1, GIM-1 e SIM-1. Os resultados podem ser obtidos em 2 horas após o isolamento de $P$. aeruginosa ${ }^{16}$.

Marra e cols ${ }^{17}$ encontraram taxas de mortalidade e morbidade entre pacientes com infecções da corrente sanguínea por P. aeruginosa produtoras de MBL do Hospital São Paulo superior a 85,7\%. Em nosso estudo, apesar de ter sido realizado apenas com pacientes do IOP-GRAACC, foi observado uma taxa de mortalidade de $42,6 \%$ sendo $70,6 \%$ nos pacientes com amostras produtoras de $b l a_{\text {SPM-1 }}$.

As opções terapêuticas para o tratamento de infecções causadas por $P$. aeruginosa são limitadas e incluem penicilinas com atividade antipseudomonas, cefalosporinas de amplo espectro, monobactâmico, carbapenêmicos, e fluoroquinolonas, particularmente a ciprofloxacina ${ }^{18}$. Para amostras multirresistentes, incluindo aos carbapenêmicos, a única opção terapêutica disponível tem sido as polimixinas (colistina e polimixina B). Apesar de raros já existem relatos de amostras também resistentes a esses antimicrobianos ${ }^{19}$. Nas amostras testadas, no presente estudo, pelo método de disco difusão, todas (100\%) foram sensíveis a polimixina B; entretanto, o $\mathrm{CSLI}^{2}$ padronizou o teste sensibilidade a polimixinas apenas para diluição em ágar. Apesar da sensibilidade ao aztreonam nas amostras produtoras de $b l a_{s p m-1}$, não utilizamos esse antimicrobiano na terapêutica dessas infecções, mas sim como marcador de possível presença de MBL, já que não é hidrolisado por essas enzimas.

Em conclusão, evidenciamos a presença de um clone de $P$. aeruginosa resistente aos carbapenêmicos carreando o gene $b l a_{\mathrm{SPM}-1}$ que persistiu no período entre novembro/2000 a dezembro/2005 em ICS de pacientes do IOP-GRAACC. Não detectamos a presença de outras metalo enzimas $b l a_{\mathrm{IMP}-1}, b l a_{\mathrm{VIM}-1}, b l a_{\mathrm{VIM}-2}$ nas amostras de $P$. aeruginosa resistentes a carbapenêmicos. A terapêutica antimicrobiana foi adequada em apenas $27,7 \%$ dos pacientes com bacteremia por $P$. aeruginosa carreando o gene $b l a_{\mathrm{SPM}-1}$. Observamos uma maior letalidade, nos pacientes com infecção causada por $P$. aeruginosa carreando o gene $b l_{\mathrm{SPM}-1}$ em relação as demais pacientes.

\section{CONFLITO DE INTERESSE}

Os autores declaram não haver nenhum tipo de conflito de interesse no desenvolvimento do estudo.

\section{REFERÊNCIAS}

1. Toleman MA, Simm AM, Murphy TA, Gales AC, Biedenbach DJ, Jones RN, et al Molecular characterization of SPM-1, a novel metallo-beta-lactamase isolated in Latin America: report from the SENTRY antimicrobial surveillance programme. J Antimicrob Chemother 2002; 50:673-679.

2. Clinical Laboratory Standards Institute (CLSI). Performance standards for antimicrobial susceptibility testing. Fifteenth Informational Supplement Approved Standard M100-S15 Wayne; 2007;

3. Gales AC, Jones RN, Turnidge J, Rennie R, Ramphal R. Characterization of Pseudomonas aeruginosa isolates: occurrence rates, antimicrobial susceptibility patterns, and molecular typing in the global SENTRY Antimicrobial Surveillance Program, 1997-1999. Clin Inf Dis 2001; 32(suppl 2):146-155.

4. Picão RC. Avaliação comparativa de diferentes testes femotípicos para a detecção de amostras produtoras de Metalo-â-lactamases. [tese de mestrado]. [São Paulo (SP)]: Escola Paulista de Medicina/Universidade Federal de São Paulo; 2007.

5. Tenover FC, Arbeit RD, Goering RV, Mickelsen PA, Murray BE, Persing DH, et al. Interpreting chromosomal DNA restriction patterns produced by Pulsed-Field Gel Electrophoresis: criteria for bacterial strain typing. J Clin Microbiol 1995; 33:2233-2239.

6. Levine AS, Schimpff SC, Graw Jr RG, Young RC. Hematologic malignancies and outher marrow failure states: Progress in the management of complicating infections. Sem Hematol 1974; 11:141-202. 
7. Bodey GP. Infection in cancer patients: A continuing association. Am J Med 1986; 81:11-26.

8. Poirel L, Magalhaes M, Lopes M, Nordmann P. Molecular analysis of metallobeta-lactamase gene bla(SPM-1)-surrounding sequences from disseminated Pseudomonas aeruginosa isolates in Recife, Brazil. Antimicrob Agents Chemother 2004; 48:1406-1409.

9. Walsh TR, Toleman MA, Hryniewicz W, Bennett PM, Jones RN. Evolution of an integron carrying blaVIM-2 in Eastern Europe: report from the SENTRY Antimicrobial Surveillance Program. J Antimicrob Chemoth 2003; 52:116-119.

10. Toleman MA, Biedenbach D, Bennett D, Jones RN, Walsh TR. Genetic characterization of a novel metallo-beta-lactamase gene, bla $_{\mathrm{IMP}-13}$, harboured by a novel Tn5051-type transposon disseminating carbapenemase genes in Europe: report from the SENTRY worldwide antimicrobial surveillance programme. J Antimicrob Chemoth 2003; 52:583-590.

11. Mendes RE, Toleman MA, Ribeiro J, Sader HS, Jones RN, Walsh TR. Integron carrying a novel metallo-beta-lactamase gene, bla $a_{\mathrm{IMP}-16}$, and a fused form of aminoglycoside-resistant gene $\operatorname{aac}\left(6^{\prime}\right)-30 / \operatorname{aac}\left(6^{\prime}\right)-I b^{\prime}$ : Report from the SENTRY Antimicrobial Surveillance Program. Antimicrob Agents Chemother 2004;48:4693-702.

12. Patzer J, Toleman MA, Deshpande LM, Kaminska W, Dzierzanowska D, Bennett PM, et al. Pseudomonas aeruginosa strains harbouring an unusual blaVIM-4 gene cassette isolated from hospitalized children in Poland (1998-2001).J Antimicrob Chemoth 2004; 53:451-456.

13. Balan APRT. Epidemiologia molecular de Pseudomonas aeruginosa produtoras de Metalo- $\beta$-lactamases isoladas de pacientes do Hospital São Paulo em 2004 [tese de mestrado]. [São Paulo (SP) ]: Universidade Federal de São Paulo/Escola Paulista de Medicina; 2007.

14. Gales AC, Menezes LC, Silbert S, Sader HS. Dissemination in distinct Brazilian regions of an epidemic carbapenem-resistant Pseudomonas aeruginosa producing SPM metallo-beta-lactamase. Journal of Antimicrobial Chemotherapy 2003; 52:699-702.

15. Marra AR. Impacto clínico das infecções da corrente sangüínea por Pseudomonas aeruginosa produtoras de metalo-â-lactamases [tese de doutorado]. [São Paulo (SP) ]: Escola Paulista de Medicina/Universidade Federal de São Paulo; 2004.

16. Mendes RE, Kiyota KA, Monteiro J, Castanheira M, Andrade SS, Gales AC, et al. Rapid Detection and Identification of Metallo-beta-Lactamase-Encoding Genes by Multiplex Real-Time PCR Assay and Melt Curve Analysis. J Clin Microbiol 2007; 45:544-547.

17. Marra AR, Pereira CAP, Gales AC, Menezes LC, Cal RGR, Souza JMA, et al. Bloodstream Infections with Metallo- $\beta$-Lactamase-Producing Pseudomonas aeruginosa: Epidemiology, Microbiology, and Clinical Outcomes. Antimicrob Agents Chemoth 2006; 50:388-390.

18. Carmeli Y, Troillet N, Eliopoulos GM, Samore MH. Emergence of antibioticresistant Pseudomonas aeruginosa: comparison of risks associated with different antipseudomonal agents. Antimicrob Agents Chemother 1999; 43:1379-1382.

19. Gales AC, Jones RN, Sader HS. Global assessment of the antimicrobial activity of polymyxin B against 54731 clinical isolates of Gram-negative bacilli: report from the SENTRY antimicrobial surveillance programme (2001-2004). Clin Microbiol Inf 2006; 12:315-21. 